\title{
Hippocampal CA2 Organizes CA1 Slow and Fast $\gamma$ Oscillations during Novel Social and Object Interaction
}

\author{
Logan Y. Brown, ${ }^{1,2}$ Georgia M. Alexander, ${ }^{1}$ Jesse Cushman, ${ }^{1}$ and ${ }^{\circledR S e r e n a ~ M . ~ D u d e k ~}{ }^{1}$
}

https://doi.org/10.1523/ENEURO.0084-20.2020

\begin{abstract}
${ }^{1}$ Neurobiology Laboratory, National Institute of Environmental Health Sciences, National Institutes of Health, Research Triangle Park, NC 27709 and ${ }^{2}$ Neuroscience Curriculum, University of North Carolina at Chapel Hill, University of North Carolina School of Medicine, Chapel Hill, NC 27599
\end{abstract}

\begin{abstract}
A key goal in hippocampal research is to understand how neuronal activity is generated and organized across hippocampal subregions to enable memory formation and retrieval. Neuronal activity in CA2 is regulated by spatial and social investigation as well as by novelty (Mankin et al., 2015; Alexander et al., 2016), and CA2 activity controls population oscillatory activity in the slow $\gamma$ and ripple ranges within hippocampus (Kay et al., 2016; Oliva et al., 2016; Boehringer et al., 2017; Alexander et al., 2018). CA2 neurons are also required for social recognition memory (Stevenson and Caldwell, 2012; Hitti and Siegelbaum, 2014; Smith et al., 2016). Because CA1 exhibits layer-specific organization (Scheffer-Teixeira et al., 2012; Lasztóczi and Klausberger, 2014, 2016) reflective of its inputs (Fernández-Ruiz et al., 2012; Schomburg et al., 2014), and because CA2 activity controls CA1 slow $\gamma$ (Alexander et al., 2018), we hypothesized that silencing CA2 would affect CA1 slow $\gamma$ in a layer-specific manner during investigation of a novel social stimulus. While recording from CA1, we leveraged molecular tools to selectively target and inhibit CA2 pyramidal cells using inhibitory DREADDs while subject mice investigated novel animals or objects. We found that CA2 inhibition reduced slow $\gamma$ power during investigation of a novel animal and fast $\gamma$ power during both novel object and animal investigation in a manner reflective of the CA2 axonal projection zones within CA1. Our results suggest that CA2 contributes to CA1 slow and fast $\gamma$ oscillations in a stimulus-specific manner.
\end{abstract}

Key words: CA2; DREADD; hippocampus; oriens; slow $\gamma$; social

\section{Significance Statement}

Using chemogenetics and electrophysiology, we show that CA2 contributes to slow $\gamma$ oscillations in CA1 during novel animal investigation and fast $\gamma$ during novel animal or object investigation in a layer-specific manner reflective of CA2 connectivity. Slow $\gamma$ oscillations in CA1 rely on CA3 and CA2 input during running, and CA2 neuronal activity is required for social memory. Our findings of reduced slow $\gamma$ oscillations in CA1 layers targeted by CA2 axons on reduction of CA2 activity support the involvement of CA2 in slow $\gamma$ oscillations, and the finding that slow $\gamma$ oscillations were impaired by CA2 silencing selectively during novel animal investigation are consistent with a role for CA2 in social cognition.

\section{Introduction}

The mammalian hippocampus is critical for the formation of long-term episodic memories (Tulving, 1984).

Received March 5, 2020; accepted March 6, 2020; First published March 20, 2020.

The authors declare no competing financial interests.
Despite the relative stability of neuronal connections post-development, neurons may be capable of functionally coupling and uncoupling to one another based on

Author contributions: L.Y.B., G.M.A., and S.M.D. designed research; L.Y.B. performed research; L.Y.B., G.M.A., and J.C. analyzed data; L.Y.B., G.M.A., and S.M.D. wrote the paper. 
their frequency characteristics (Buzsáki and Draguhn, 2004). Although oscillations detected in the local field potential (LFP) are highly structured across brain areas, the precise patterns of synchronization change dynamically with behavior.

Rodent hippocampal oscillations are dominated by three frequencies of activity: theta $(\sim 6-9 \mathrm{~Hz}$; Buzsáki et al., 2003), $\gamma(\sim 25-100 \mathrm{~Hz}$; Colgin, 2016), and ripples ( $\sim 100-200 \mathrm{~Hz}$; Csicsvari et al., 1999). $\gamma$ Can be further divided into slow $(\sim 25-55 \mathrm{~Hz})$ and fast $(\sim 55-100 \mathrm{~Hz})$ bands. These two frequencies are thought to be generated through separate mechanisms and play distinct roles in memory (Colgin et al., 2009). Slow $\gamma$ in CA1 is prevalent in stratum radiatum (SR; Schomburg et al., 2014), where CA3 neuronal projections predominate, and are coherent with slow $\gamma$ in CA3 (Colgin, 2015). Further, silencing of CA3 synaptic output with tetanus toxin light chain reduces slow $\gamma$ power in CA1 by $\sim 30 \%$ (Middleton and McHugh, 2016). CA2 has also been shown to contribute to slow $\gamma$ in CA1, as chemogenetic manipulation of CA2 activity bi-directionally modulates CA1 slow $\gamma$ power during running (Alexander et al., 2018). In contrast, fast $\gamma$ oscillations are prevalent in stratum lacunosum moleculare (SLM) of CA1 (Schomburg et al., 2014), co-occur with fast $\gamma$ in medial EC (Colgin et al., 2009), and are impaired by lesioning of medial EC (Bragin et al., 1995), supporting the notion that CA1 fast $\gamma$ relies on medial EC activity. Fast $\gamma$ is thought to contribute to memory encoding because fast $\gamma$ power is increased there during an animal's investigation of novel stimuli (Kemere et al., 2013; Zheng et al., 2016).

Pyramidal neurons in area CA2 strongly express a number of genes, including Amigo2 (Lein et al., 2007; Laeremans et al., 2013), PCP4 (San Antonio et al., 2014), RGS14 (Lee et al., 2010), and others (Dudek et al., 2016). Neurons in CA2 receive excitatory input from the EC and CA3 (Chevaleyre and Siegelbaum, 2010; Kohara et al., 2014) and, in turn, project to a variety of intra- and extrahippocampal targets, but primarily target CA1 stratum oriens (SO) and deep pyramidal cells (Tamamaki et al., 1988; Kohara et al., 2014). Neurons in CA2 also project to CA1 SR to a lesser extent, but not to SLM. Several studies have investigated the function of CA2 in vivo, implicating it in the organization of sharp wave ripple (Kay et al., 2016; Oliva et al., 2016) and slow $\gamma$ oscillations (Alexander et al., 2018), as well as social memory (Stevenson and Caldwell, 2012; Hitti and Siegelbaum, 2014; Alexander et al., 2016;

This work was supported by the Intramural Research Program of the National Institute of Environmental Health Sciences, National Institutes of Health Grant Z01 ES100221.

Acknowledgements: We thank the National Institute of Environmental Health Sciences Fluorescence Microscopy and Imaging Center and the animal care staff for their support. We also thank Dr. Paul Manis, Dr. Flavio Frohlich, and Dr. Bernd Gloss.

Correspondence should be addressed to Serena M. Dudek at dudek@niehs. nih.gov.

https://doi.org/10.1523/ENEURO.0084-20.2020

Copyright (C) 2020 Brown et al.

This is an open-access article distributed under the terms of the Creative Commons Attribution 4.0 International license, which permits unrestricted use, distribution and reproduction in any medium provided that the original work is properly attributed.
Smith et al., 2016). However, it currently remains unknown whether CA2 coordinates hippocampal oscillations during social behaviors.

Given the evidence for CA2's role in social memory and that CA1 $\gamma$ oscillations are regulated in a layer-specific manner reflective of its upstream inputs (Fernández-Ruiz et al., 2012; Scheffer-Teixeira et al., 2012; Lasztóczi and Klausberger, 2014, 2016), we predicted that if CA2-dependent oscillations in CA1 contribute to its function in social memory, then they should occur during social exposure and, given the distribution of CA2 axons in CA1, should be organized in a layer-specific manner. Thus, we asked whether CA1 $\gamma$ oscillations also rely on CA2 output during investigation of novel social stimuli. We recorded from each layer of CA1 in adult, male Amigo2-icreERT2 mice while mice investigated a novel social or object stimulus and then inhibited CA2 pyramidal cells using human-muscarinic-4-DREADD-Gi (hM4Di) to test whether CA2 contributes to $\gamma$ oscillations in CA1 during novel object and social stimulation.

\section{Materials and Methods}

\section{Animals}

Experiments were conducted in adult male (more than eight-week-old) Amigo2-icreERT2 mice. Mice were housed under a 12/12 h light/dark cycle with ad libitum access to food and water. Mice were naive to any treatment, procedure or testing at the beginning of the experiments described here. Mice were group-housed until the time of virus infusion, at which point they were singly housed. All procedures were approved by the Institute's Animal Care and Use Committee and were in accordance with the National Institutes of Health guidelines for care and use of animals.

\section{Viruses}

All AAV viruses were produced by the Gene Therapy Center Vector Core at the University of North Carolina at Chapel Hill and had titers of $>10^{12}$ genome copies $/ \mathrm{ml}$. For chemogenetic manipulation using hM4Di-mCherry, mice were bilaterally infused with $0.5 \mu \mathrm{l}$ of AAV5-hSynDIO-hM4Di-mCherry into each hemisphere of the brain.

\section{Stereotaxic virus infusions and tamoxifen treatment}

For virus-infusion surgery, mice were anesthetized with ketamine $(100 \mathrm{mg} / \mathrm{kg}$, i.p.) and xylazine $(7 \mathrm{mg} / \mathrm{kg}$, i.p.), then placed in a stereotaxic apparatus while on a heated pad. Sedation was maintained with 1.5-2.5\% isoflurane during surgery if animals regained reflex responses at any point during the surgery. Following three swabs with betadine and saline rinse, an incision was made down the midline of the scalp, a burr hole was drilled above each target region and viruses were microinjected using a 1- $\mu$ l Neuros Hamilton syringe at a rate of $100 \mathrm{nl} / \mathrm{min}$. Following infusion, the needle was left in place for five additional minutes to allow for diffusion of the virus before slowly being retracted. Injection coordinates for CA2 were $-2.30 \mathrm{~mm}$ AP, $\pm 2.50 \mathrm{~mm} \mathrm{ML}$, and $-1.90 \mathrm{~mm}$ DV from bregma. The scalp was then sutured shut and animals were administered buprenorphine $(0.1 \mathrm{mg} / \mathrm{kg}$, s.c.) for pain and returned to their cages. Two weeks following AAV infusion, Amigo2- 
icreERT2 mice began daily tamoxifen intraperitoneal injections $(100 \mathrm{mg} / \mathrm{kg}$ tamoxifen dissolved in warmed corn oil) for a total of $7 \mathrm{~d}$. Animals were allowed to recover from tamoxifen treatment for one week before undergoing electrode implantation to allow sufficient time for estrogen receptor translocation to the nucleus and subsequent recombination.

\section{Electrode implantation}

At least one week after the last tamoxifen treatment, mice for in vivo electrophysiology were implanted with NeuroNexus A1x32 silicone electrodes (NeuroNexus). Mice were anesthetized with ketamine $(100 \mathrm{mg} / \mathrm{kg}$, i.p.) and xylazine $(7 \mathrm{mg} / \mathrm{kg}$, i.p.), then placed in a stereotaxic apparatus while on a heated pad. An incision was made in the scalp, and the skull was cleaned and dried. A screw for the electrode reference wire was positioned $\sim 4 \mathrm{~mm}$ posterior and $2 \mathrm{~mm}$ lateral to bregma over the right cortical hemisphere and a screw for the ground wire was positioned in the skull over the cerebellum. Three additional anchor screws were secured to the skull. Electrodes were then lowered into the hole drilled over dorsal CA1: -1.30 $\mathrm{mm}$ AP, $-1.40 \mathrm{~mm} \mathrm{ML},-1.85 \mathrm{~mm}$ DV from bregma, and the reference and ground wires were attached to their respective screws. The NeuroNexus A1x32 electrode is designed with 32 recording sites arranged linearly along a single shank, spaced $25 \mu \mathrm{m}$ apart, providing a tissue coverage range of $775 \mu \mathrm{m}$.

\section{Recording protocol}

We recorded one session per day for eight sequential days. Treatment with clozapine-N-oxide (CNO) or vehicle alternated across days, as did the type of stimulus presented (animal or object). All recordings were made during the animal's light phase under red light illumination to encourage exploration. For these experiments, animals were allowed to freely explore a clear Plexiglas cage (40.5 $\times 24.5 \mathrm{~cm}$ ). Bedding was placed in the bottom of the cage (as in home cages) to encourage exploration. Curtains surrounded the cage to prevent viewing of the experimental room and limit light exposure. The recording protocol (Fig. $1 A$ ) proceeded as follows: 40 min before the start of recording, subjects were administered CNO $(3.0 \mathrm{mg} / \mathrm{kg})$ or saline and returned to their home cages outside the recording room. Ten minutes before the start of the experiments, subjects were lightly anesthetized with isoflurane to allow attachment of wireless head stages and were transferred to the recording room where room lights were turned off and red lights were illuminated. Five minutes before the start of recording, subjects were transferred from their home cage to the recording cage and allowed to habituate for $5 \mathrm{~min}$ before the start of recording. For the first $5 \mathrm{~min}$ of each recording session, no stimulus was present. This phase was used as a baseline period to assess distance traveled and time spent mobile. For the second 5 min of each recording session either a novel animal or novel object was inserted into the recording cage in a wire mesh cup. The novel animal stimulus (Fig. 1B) was a male, juvenile conspecific from the Amigo2-
icreERT2 line, naive to any experimental testing before being used in the test session. The novel object stimulus (Fig. 1C) consisted of inanimate wooden blocks (Kaytee Pet Supplies), which varied in color and shape, measured $\sim 2 " \times 2$ " $\times 2$ ", and had a cedar scent. In each session, two to three blocks were stacked together and used as the object stimulus. Periods in which a subject was actively investigating the stimulus were isolated and used for spectral analysis.

\section{Neurophysiological data acquisition and behavioral tracking}

Neural activity was transmitted via a 32-channel wireless $10 \times$ gain head stage (Triangle BioSystems International) and was acquired using the Cerebus acquisition system (Blackrock Microsystems). Continuous LFP data were bandpass filtered at $0.3-500 \mathrm{~Hz}$ and stored at $1000 \mathrm{~Hz}$. For behavioral tracking, videos of recording sessions were acquired with a sampling rate of $30 \mathrm{~Hz}$ using the Neuromotive Software (Blackrock Microsystems) and an infrared camera. Behavior was analyzed offline using the Ethovision XT (Noldus) behavioral tracking software. Subjects' smoothed instantaneous velocity, measured from reflective tape on the headstage, was used to isolate periods of running from periods of relative immobility. Samples in which the animal's smoothed instantaneous velocity exceeded a threshold of $\geq 7 \mathrm{~cm} / \mathrm{s}$ were designated as periods of running and excluded from analysis. The $7-\mathrm{cm} / \mathrm{s}$ threshold was selected based on manual optimization of tracking parameters to best divide periods of quiet immobile rest from active traversing of the environment. For selection of periods in which subjects were actively investigating stimuli, a virtual $10-\mathrm{cm}$ circle was drawn around the wire mesh cup in which stimuli were presented, and periods in which the subject's body, nose, or tail were located within the circle were isolated (Fig. 1B,C). For spectral analyses, only samples in which subjects were both immobile and located inside the virtual circle were used. Because the animal was not traversing the environment during the periods used for our LFP analyses, a prominent peak in the theta range was not expected. All indexed tracking information was exported from Ethovision and then used to select the appropriate LFP samples from behavioral periods of interest in MATLAB.

\section{Neurophysiological data analysis}

All neuronal data analyses were performed with MATLAB version R2016b (MathWorks) using custom written scripts for (1) selection of LFP samples corresponding to periods in which subjects performed behaviors of interest, (2) artifact rejection, and (3) spectral analysis of LFP samples using a family of Morlet wavelets. First, behavioral vectors were generated from tracking data generated by Noldus Ethovision XT to designate periods in which subjects were both immobile and inside the virtual circle drawn around the stimulus. The behavioral vectors were then used to match periods in which animals performed behaviors of interest to the appropriate LFP samples. Second, noise and muscle artifacts were removed from recordings based on a 6-standard deviation 


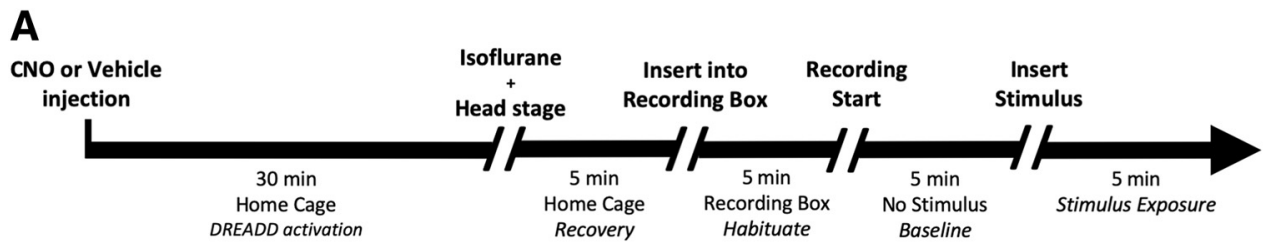

B

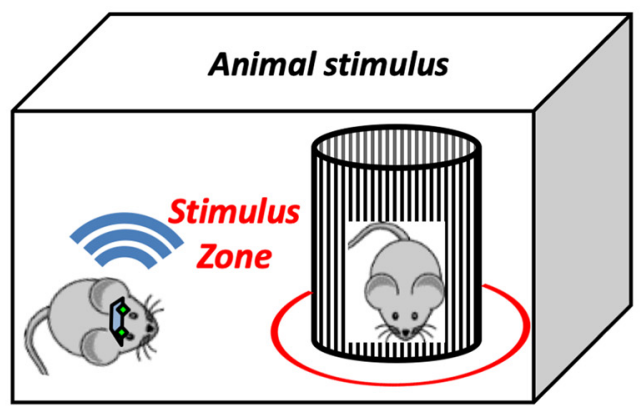

C

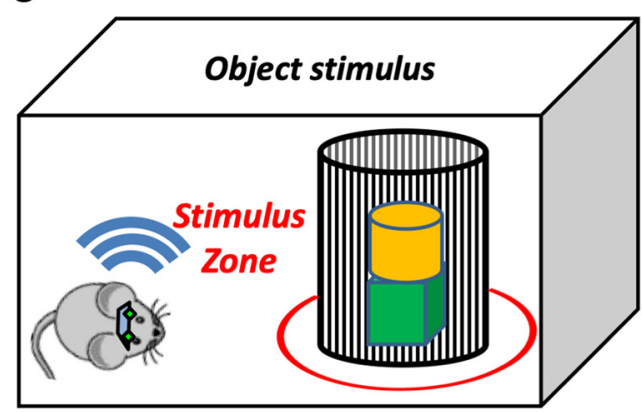

Figure 1. Experimental design. A, Recording protocol used for each experimental recording session. B, Behavioral tracking for selection of periods to analyze. A virtual $10-\mathrm{cm}$ circle was drawn around the wire mesh cup and designated as the stimulus zone. Periods in which the subject's body, nose, or tail were located within the stimulus zone were isolated and used for subsequent spectral analysis.

rejection threshold; when detected, a 3-s window around the noise threshold crossing was removed. Third, spectral analysis was performed using a family of 80-wavelet carrier frequencies which increased logarithmically and had a lowfrequency cutoff of $0.5 \mathrm{~Hz}$ and a high-frequency cutoff of $100 \mathrm{~Hz}$. For each recording session, the time-frequency representation of the data were obtained by convolving the LFP with the family of wavelets to produce a vector of the same length as the original LFP data.

Over the eight recording days, each of the four-possible drug and stimulus combinations (vehicle-animal, vehicleobject, CNO-animal, CNO-object) were delivered twice, and the LFP power values from these two repeat sessions were averaged. For statistical analysis of LFP power we focused on the theta $(6-9 \mathrm{~Hz})$, slow $\gamma(25-55 \mathrm{~Hz})$, and fast $(55-100 \mathrm{~Hz}) \gamma$ frequencies ranges. An average was calculated for each frequency band separately by averaging all wavelet values that were used to generate the time-frequency representation of that band. For example, the slow $\gamma$ band was computed using 19 wavelets, so these 19 values were averaged to yield a single value for the slow $\gamma$ band. These values were calculated for each hippocampal layer in each subject and used for group comparisons of power under the different experimental conditions.

Ripple oscillations, paired with immunohistochemical (IHC) methods (described below), were used to identify electrode placement. For ripple measurements, periods in which subjects were immobile were isolated and the LFPs from these periods were filtered in the ripple range (100$300 \mathrm{~Hz}$ ). The channel with the maximum amplitude of putative ripple events was identified as the pyramidal cell layer (Fig. 2G).

\section{IHC and electrode localization}

To aid in electrode localization, at the end of all experiments for each animal, a small amount of current was passed through the channel corresponding to the bottom-most electrode pad of each electrode ( $5 \mu \mathrm{A}$ for $5 \mathrm{~s}$ ) to create a small lesion at the most ventral recording location of that probe, visible on subsequent IHC processing. Three hours after creating the lesion, animals were euthanized with Fatal Plus (sodium pentobarbital, $50 \mathrm{mg} / \mathrm{ml}$; $>100 \mathrm{mg} / \mathrm{kg}$ ) and underwent transcardial perfusion with $4 \%$ paraformaldehyde. Heads with electrodes remaining implanted were submerged in $4 \%$ paraformaldehyde for $72 \mathrm{~h}$. Brains were then carefully removed, rinsed in PBS, and sectioned at $40 \mu \mathrm{m}$ on a vibratome.

For IHC, brain sections were rinsed in PBS, boiled in deionized water for $3 \mathrm{~min}$, and blocked for at least $1 \mathrm{~h}$ in 3$5 \%$ normal goat serum/0.01\% Tween 20 PBS. Sections were then incubated in mouse anti-RGS14 (UC Davis, 75170, 1:250) and rabbit anti-WFS1 (ProteinTech, 11558$1-A P, 1: 250)$ primary antibodies. Sections in which an electrode track was visible were incubated in rabbit anti-IBA1 (Wako, catalog 012-26 723, 1:500), a marker for activated microglia (Fig. 2H). Antibodies were diluted in blocking solution and sections were incubated for $24 \mathrm{~h}$. After several rinses in PBS/Tween, sections were either incubated in the secondary antibodies Alexa Fluor goat anti-mouse 488 (1:250, Invitrogen) and Alexa Fluor goat anti-rabbit 633 (1:250, Invitrogen) or Alexa Fluor goat anti-rabbit 488 (1:500, Invitrogen) for 2 h. Finally, sections were washed in PBS/Tween and mounted with Vectashield hardset mounting medium with DAPI (Invitrogen). Images of whole-brain sections were acquired with a slide scanner using the Aperio Scanscope FL Scanner (Leica Biosystems Inc.). The slide scanner uses a monochrome TDI line-scan camera, with a PC-controlled mercury light source to capture high resolution, seamless digital fluorescent images. Images of selected hippocampi were acquired on a Zeiss 780 meta confocal microscope using a $40 \times$ oil-immersion lens. 
A

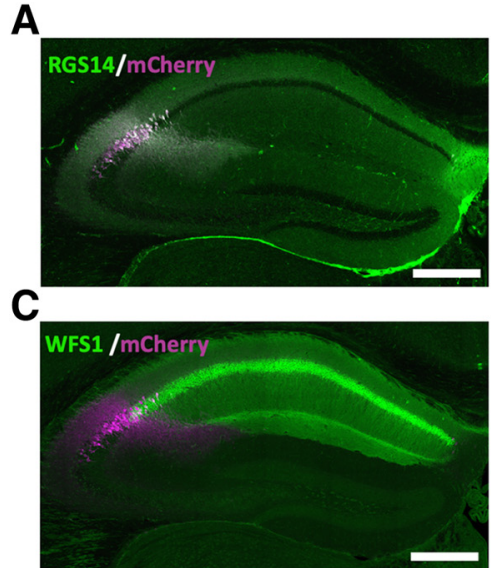

E

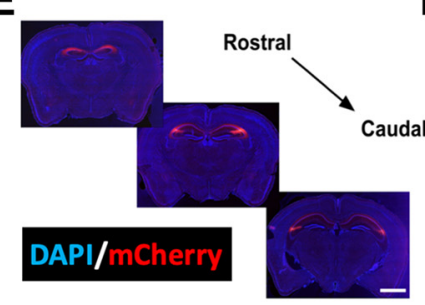

Bi
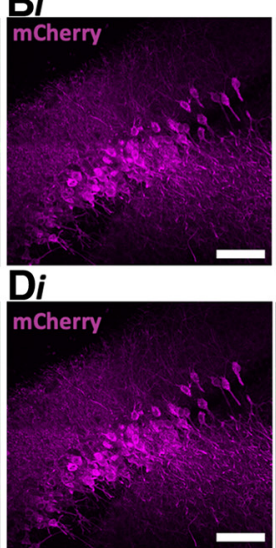

$\mathbf{F}$

G
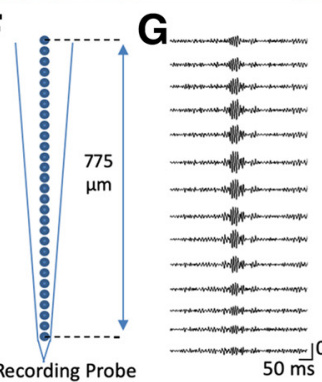

Bii

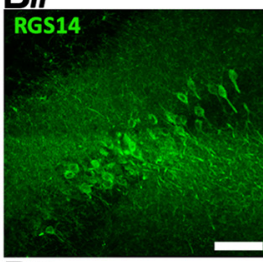

Dii

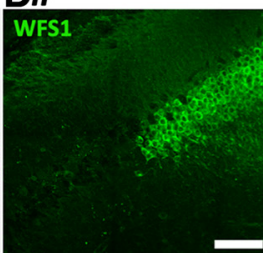

H

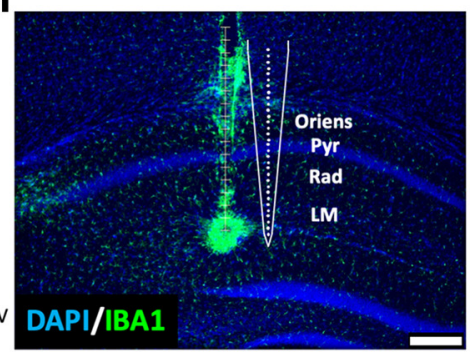

Figure 2. Selective targeting of CA2 for Gi-DREADD expression and CA1 for recording. Amigo2-icreERT2 mice were infused bilaterally with AAV-hSyn-DIO-hM4Di-mCherry, treated with tamoxifen, and implanted with NeuroNexus A1x32 silicone probes. $\boldsymbol{A}$, Co-expression of Cre-dependent hM4Di-mCherry and RGS14, a marker for CA2 pyramidal cells, in the hippocampus. B, High magnification image showing the expression pattern of (i) hM4Di-mCherry in CA2, (ii) RGS14 in CA2, and (iii) merged images. No evidence of hM4Di-mCherry expression was found outside of the RGS14-expressing region. $\boldsymbol{C}$, Expression of Cre-dependent hM4Di-mCherry and WFS1, a marker for CA1 pyramidal cells, across the hippocampus. $\boldsymbol{D}$, High-magnification image showing the expression pattern of (i) hM4Di-mCherry in CA2, (ii) lack of WFS1 in CA2, and (iii) merged images. No evidence of mCherry colocalization with WFS1 was observed. $\boldsymbol{E}$, Expression pattern of hM4Di-mCherry across the rostral to caudal extent of CA2. $\boldsymbol{F}$, Schematic diagram of NeuroNexus A1x32 probe detailing probe dimensions. G, Ripple-filtered $(100-300 \mathrm{~Hz})$ LFPs across the CA1 layers. The channel with the maximal amplitude of putative ripples was designated as located in the pyramidal cell layer. $\boldsymbol{H}$, At the end of experiments, an electrolytic lesion was made on the bottom channel of the electrode and sections were processed by IHC to highlight IBA1, a marker for activated microglia. Representative recording locations in CA1 were reconstructed based on IBA1 staining and putative ripple oscillations. Scale bars $=200 \mu \mathrm{m}(\boldsymbol{A}, \boldsymbol{C}, \boldsymbol{H}), 50 \mu \mathrm{m}(\boldsymbol{B}, \boldsymbol{D})$, and $1 \mathrm{~mm}(\boldsymbol{E})$.

\section{Sample size and group assignment}

For all experiments presented, 19 male Amigo2-icreERT2 (10 Amigo2-icreERT2+ and nine Amigo2-icreERT2-) mice were used. Amigo2-icreERT2 mice were arbitrarily selected from individual litters. No specific method of randomization was used to assign groups. Whenever possible, Cre+ and $\mathrm{Cre}-$ mice from the same litter were used. Animals were housed with same-sex littermates during weaning and individually housed for the duration of experiments following virus infusion. Pre-established criteria for excluding mice from analysis included (1) missed electrode implantation, (2) equipment failures during testing, and (3) insufficient investigation of experimental stimuli, with a minimum of $10 \mathrm{~s}$ of investigation per recorded session required to be included in the analysis.

\section{Statistical analyses and results reporting}

For each experiment presented in Results, the number of replicates is presented as $N$ when indicating the number of animals that were used for the experiment. Statistical tests used for each experiment are presented in the text. Statistical significance was based on $p=0.05$. All error bars in graphs represent SEM. For statistical analysis of $\gamma$ oscillatory powers presented in Figures 3, 4, we used a repeated-measures general linear model (RM-GLM; SPSS version 21, IBM) to explore potential effects of recording layer (SO, SR, pyramidal, SLM), frequency (slow $\gamma$, fast $\gamma$ ), stimulus type (animal, object), and drug (vehicle, CNO). The GLM revealed a significant interaction between the layer, frequency, and drug conditions and, based on these results, we performed a series of RM, two-tailed, paired $t$ tests to explore the source of the observed interaction effect. Statistical analysis of theta powers was performed separately from $\gamma$ oscillatory powers due to the larger values and greater variability in theta power we observed during non-mobile investigative behaviors. Theta powers for each layer were compared between vehicle and $\mathrm{CNO}$ conditions using paired $t$ tests. Locomotor velocity during LFP sampling and follow-up analyses of $\gamma$ powers on presentation of each stimulus separately (Figs. 5, 6) were analyzed using two-way ANOVAs, with Sidak post hoc tests, where appropriate. For behavioral investigation time data, we used two-tailed paired $t$ tests. For locomotor 


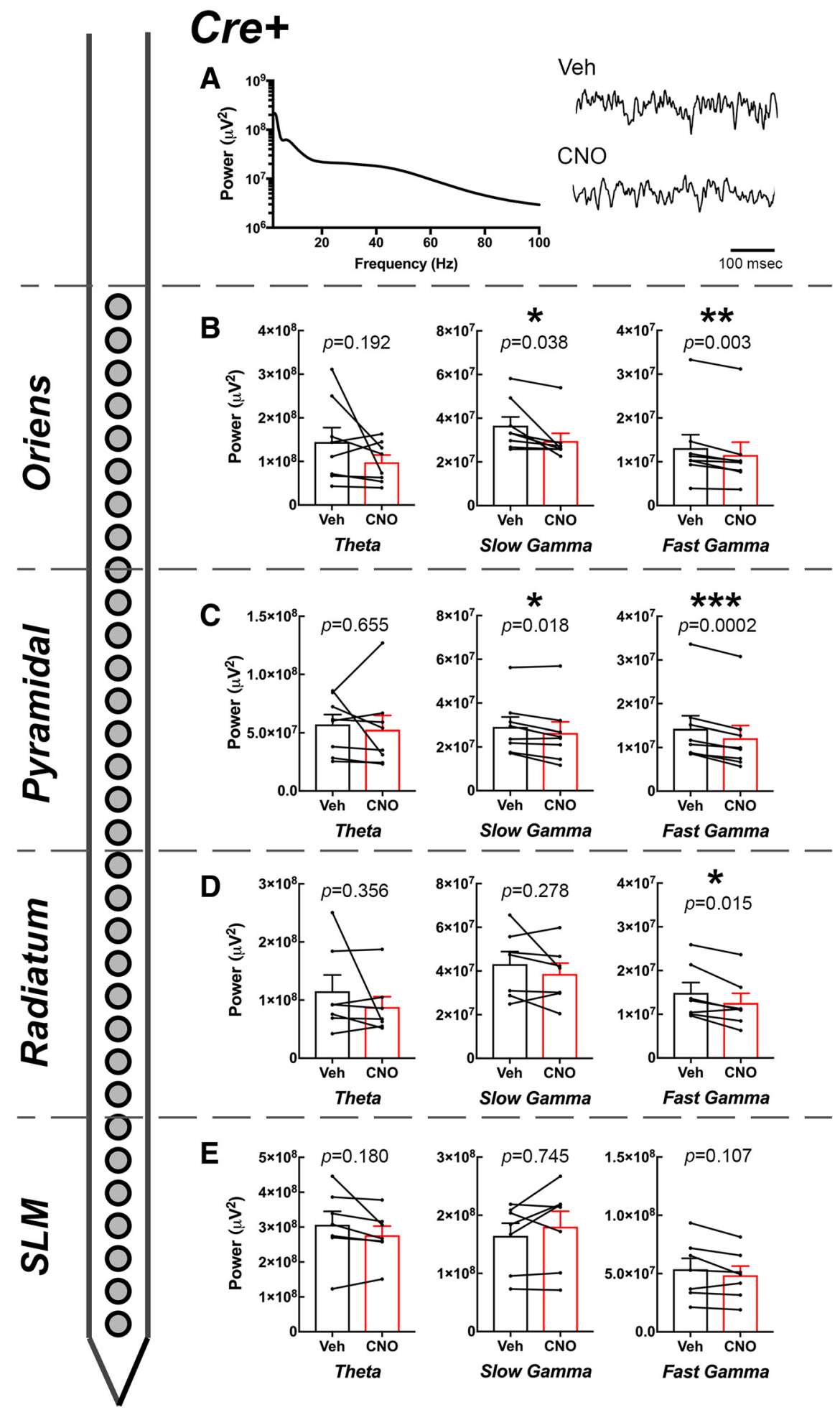

Figure 3. Effect of CNO on CA1 oscillatory power by layer in Amigo2-icreERT2 + animals. $\boldsymbol{A}$, Example PSD from the pyramidal cell layer of an Amigo2-icreERT2+ animal and raw LFP traces collected following either vehicle or CNO $(3 \mathrm{mg} / \mathrm{kg}) \mathrm{treatment}$. $\boldsymbol{B}-\boldsymbol{E}$, Theta, slow $\gamma$, and fast $\gamma$ powers measured from each layer of CA1 as indicated by the schematized shank electrode and layer of CA1 listed on the left. For each graph, population means and SEMs are represented as colored bars (black = vehicle; red $=$ CNO), and dots connected by lines represent data from individual animals for each treatment. Specific $p$ values from paired $t$ tests are shown over each graph; $* p<0.05, * * p<0.01, * * * p<0.001$. 


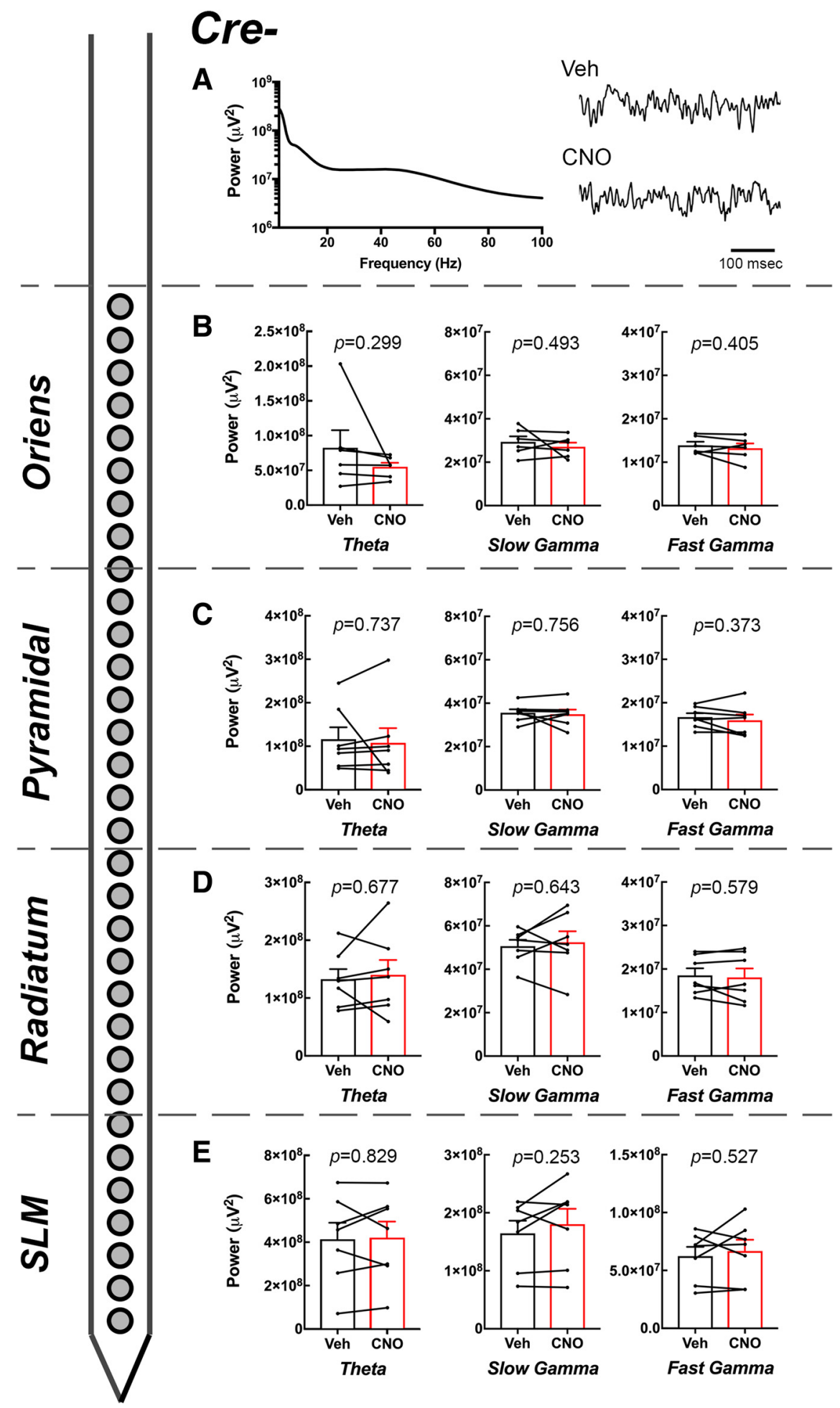

Figure 4. Effect of CNO on CA1 oscillatory power by layer in Amigo2-icreERT2- animals. A, Example PSD from the pyramidal cell layer of an Amigo2-icreERT2- animal and raw LFP traces collected following either vehicle or CNO (3 mg/kg) treatment. B-E, Theta, slow $\gamma$, and fast $\gamma$ powers measured from each layer of CA1 as indicated by the schematized shank electrode and layer of CA1 listed on the left. For each graph, population means and SEMs are represented as colored bars (black = vehicle; red = CNO), and dots connected by lines represent data from individual animals for each treatment. No comparisons were significantly different between vehicle and CNO treatment, as indicated by the specific $p$ values for each statistical analysis. Population means and SEMs are represented as colored bars (black = vehicle; red $=\mathrm{CNO}$ ), and dots connected by lines represent data from individual animals for each treatment. 


\section{Cre+}

Slow Gamma

$\frac{9}{5}$

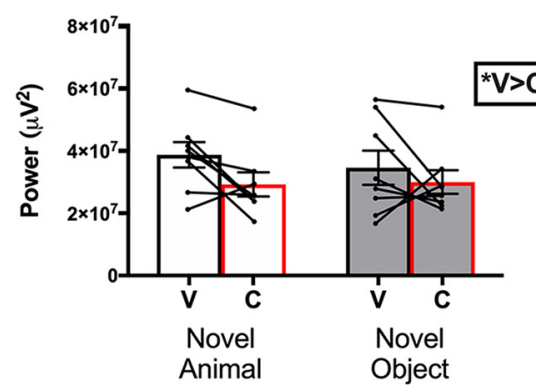

$\frac{\sqrt{0}}{\frac{\pi}{8}}$
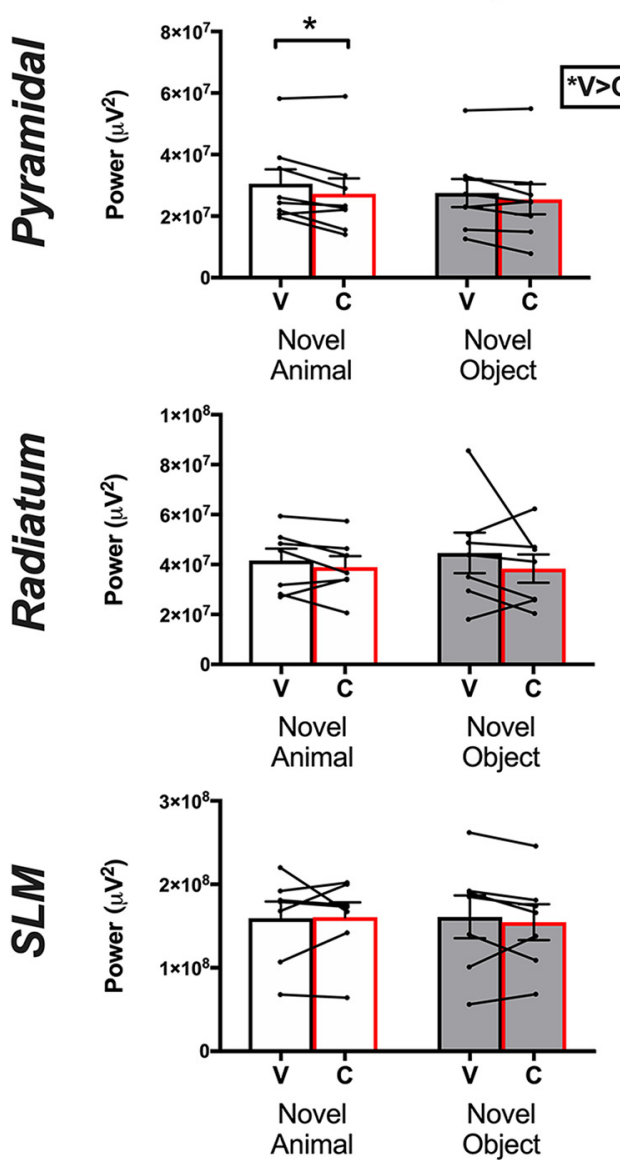

Fast Gamma

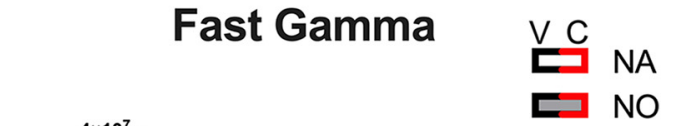

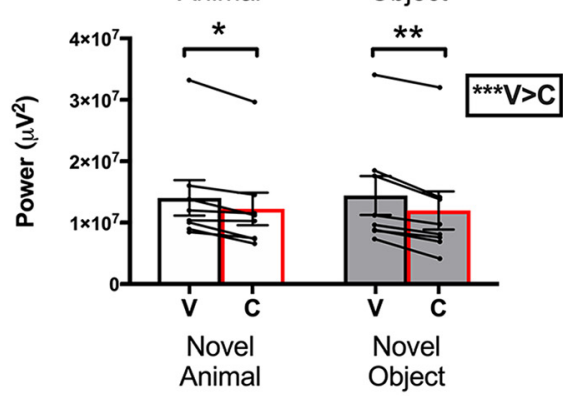
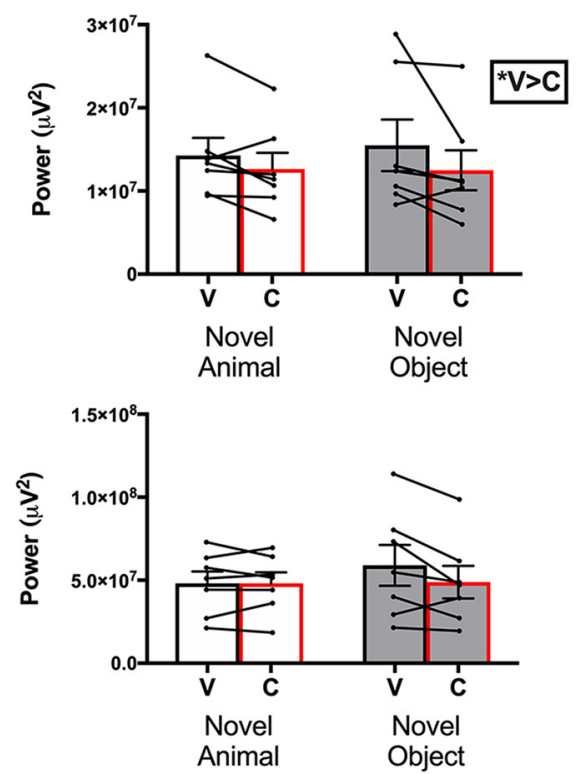

Figure 5. $\gamma$ Powers collected from Amigo2-icreERT2+ animals. Slow (left column) and fast (right column) $\gamma$ powers for each layer in CA1 following either vehicle $(V$, black bars) or CNO $(C$, red bars) treatment and on investigation of either a novel animal or a novel object. Two-way ANOVAs were performed within each layer and frequency. A main effect of treatment was found for slow and fast $\gamma$ within each of oriens and the pyramidal cell layers as well as for fast $\gamma$ in radiatum, as reported by the boxed-in result to the right of the associated graph. Population means and SEMs are represented as colored bars (black = vehicle, red = CNO and for each treatment, empty bars = novel animal, filled bars = novel object), and dots connected by lines represent data from individual animals for each treatment. Results of post hoc tests are shown on the graphs where significant; $* p<0.05, * * p<0.01, * * * p<0.001$.

data, we used RM one-way ANOVAs with GeisserGreenhouse correction for unequal variance.

\section{Results}

\section{Inhibitory DREADD expression in CA2}

To gain selective genetic access to molecularly-defined CA2, we used a previously-generated tamoxifen-inducible,
Cre-dependent mouse line, Amigo2-icreERT2 (Alexander et al., 2018). Infusion of AAVs encoding Cre-dependent hM4Di (Fig. 2A-E) with the neuron-specific human synapsin promoter into Amigo2-icreERT2 + mice allowed for selective expression of hM4Di-mCherry in CA2 pyramidal neurons without expression in other hippocampal subregions or outside of the hippocampus, as indicated by colocalization of mCherry with RGS14 (Fig. $2 A, B$ ) and lack of 


\section{Cre-}

Slow Gamma
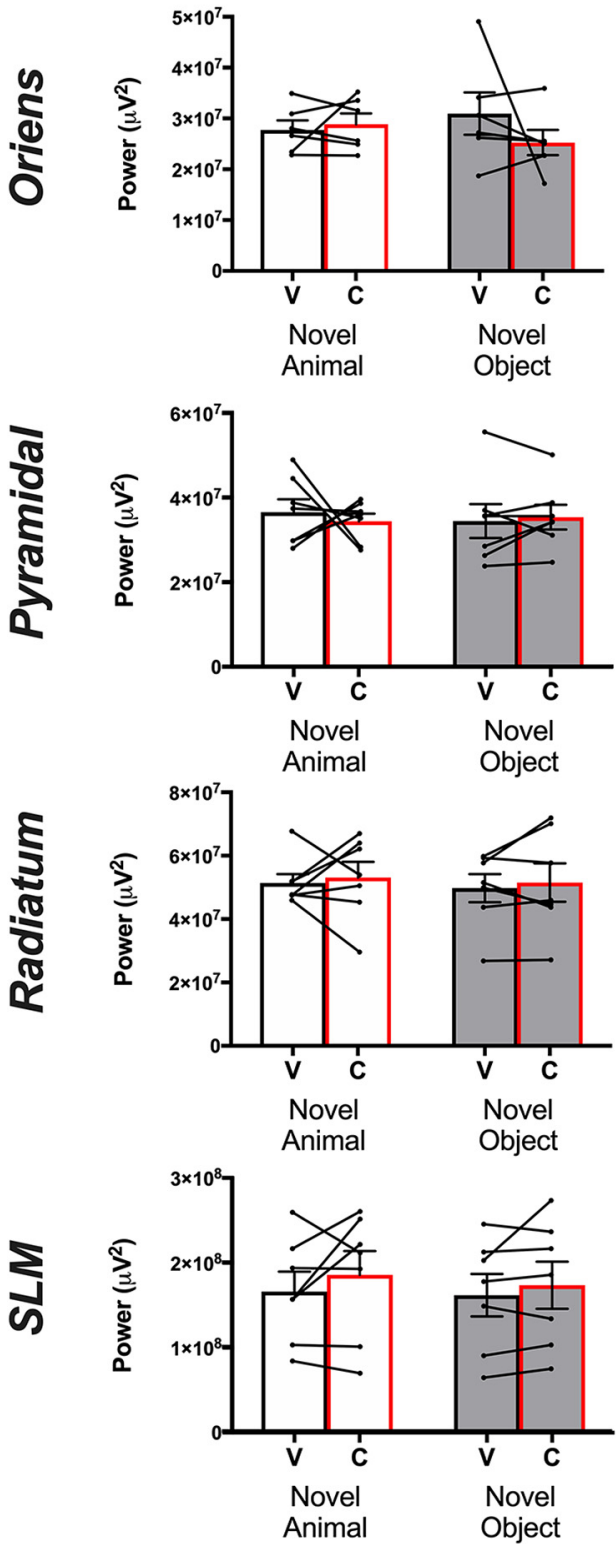

Fast Gamma
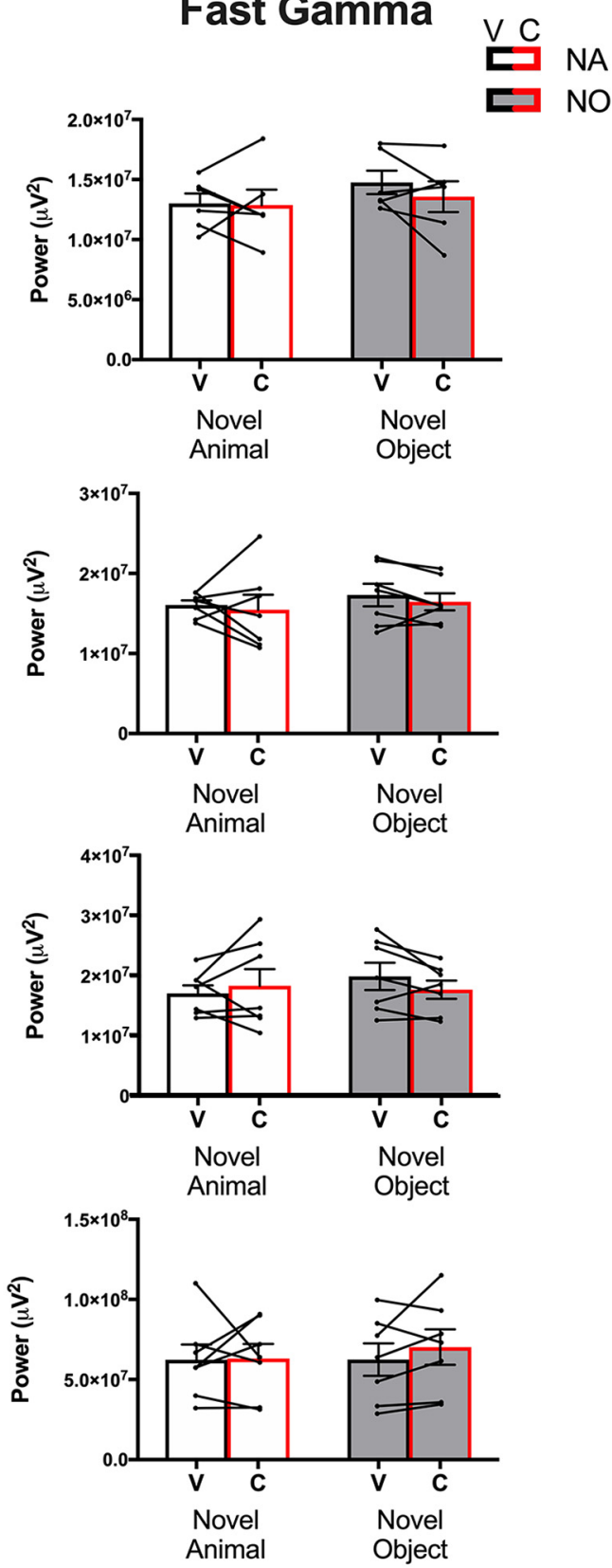

Figure 6. $\gamma$ Powers collected from Amigo2-icreERT2- animals. Slow (left column) and fast (right column) $\gamma$ powers for each layer in CA1 following either vehicle $(V$, black bars) or CNO $(C$, red bars) treatment and on investigation of either a novel animal or a novel object. Two-way ANOVAs were performed within each layer and frequency. No main effects of treatment were found for slow or fast $\gamma$ within any layer on investigation of either stimulus. Population means and SEMs are represented as colored bars (black $=$ vehicle, red = CNO and for each treatment, empty bars = novel animal, filled bars = novel object), and dots connected by lines represent data from individual animals for each treatment.

co-localization with WFS1 (Fig. 2C,D). mCherry expression was undetectable in control Amigo2icreERT2- mice infused with hM4Di-mCherry (data not shown). This mouse line enabled us to selectively target and inhibit activity of CA2 pyramidal neurons in vivo and measure the resulting network and behavioral effects.

\section{CA1 $\gamma$ oscillatory power varies by layer, frequency, and CA2 activity}

Inhibition of CA2 via expression and activation of hM4Di in CA2 pyramidal cells of Amigo2-icreERT2+ mice has been shown to reduce slow $\gamma$ power in the pyramidal cell layer of CA1 during running (Alexander et al., 2018). 
Table 1: $F$ statistics and $p$ values from the RM, GLM used to assess Amigo2-icreERT mice for effects of recording layer, frequency, drug condition, and stimulus type on oscillatory power

\begin{tabular}{|c|c|c|c|c|}
\hline \multirow[b]{2}{*}{ Source } & \multicolumn{2}{|c|}{ Cre+ } & \multicolumn{2}{|c|}{ Cre- } \\
\hline & $F$ statistics & $p$ value & $F$ statistics & $p$ value \\
\hline Frequency & 214.33 & $\overline{0.001}$ & 57.98 & $\overline{0.001}$ \\
\hline Stimulus & 0.02 & 0.887 & 0.01 & 0.948 \\
\hline Layer $\times$ frequency & 45.2 & 0.001 & 25.11 & 0.001 \\
\hline Layer $\times$ drug & 0.25 & $\overline{0.86}$ & 0.41 & $\overline{0.748}$ \\
\hline Layer $\times$ stimulus & 0.08 & $\overline{0.973}$ & 0.01 & 0.999 \\
\hline Frequency $\times$ stimulus & 0.69 & 0.439 & 0.8 & 0.411 \\
\hline Layer $\times$ frequency $\times$ stimulus & 1.06 & 0.389 & 0.56 & 0.652 \\
\hline Drug $\times$ stimulus & 0.36 & 0.571 & 0.3 & 0.606 \\
\hline Layer $\times$ drug $\times$ stimulus & 0.89 & 0.465 & 0.55 & 0.654 \\
\hline Frequency $\times$ drug $\times$ stimulus & 0.16 & 0.701 & 0.16 & 0.708 \\
\hline
\end{tabular}

Results that reached statistical significance based on $p=0.05$ are bolded and underlined.

Therefore, we tested whether inhibition of CA2 in the same manner would cause layer-specific changes in slow and fast $\gamma$ power detected in CA1 during investigation of different stimuli. Amigo2-icreERT2+ and control Amigo2icreERT2- mice infused bilaterally with hM4Di were implanted with 32-channel NeuroNexus silicone probes (Fig. 2F). For each recording session, Amigo2-icreERT2+ and control Amigo2-icreERT2- mice were treated with $3.0 \mathrm{mg} / \mathrm{kg}$ of $\mathrm{CNO}$ or an equivalent volume of saline 40 min before the start of recording. For statistical analyses, we used a RM-GLM to explore potential effects of recording layer (SO, SR, pyramidal, SLM), frequency (slow $\gamma$, fast $\gamma$ ), stimulus type (novel animal, novel object), and drug condition (vehicle, CNO), and results are presented in Table 1.

In Amigo2-icreERT2 + mice, we found significant main effects of layer $\left(F_{(1,3)}=25.31, p=0.000 ; N=8\right)$ and frequency $\left(F_{(1,1)}=214.33, p=0.000 ; N=8\right)$ as well as an interaction effect between layer and frequency $\left(F_{(1,3)}=\right.$ 45.20, $p=0.000 ; N=8$ ). Additionally, we found a significant interaction among layer, frequency, and drug $\left(F_{(1,3)}=\right.$ 3.58, $p=0.034 ; N=8$ ). By contrast, the RM-GLM revealed no main effect of stimulus $\left(F_{(1,1)}=0.02, p=0.887 ; N=8\right)$ nor any significant interactions involving stimulus. Nevertheless, given our a priori hypothesis that inhibition of CA2 may alter $\gamma$ oscillatory power in a stimulus-specific manner, we followed up by separating the data according to stimulus and compared $\gamma$ power on either vehicle or CNO treatment for each stimulus exposure (Figs. 5, 6; and described below).

\section{Inhibition of CA2 decreases CA1 slow and fast $\gamma$ power in a layer-specific manner but does not affect theta power}

Because we found no main or interaction effects of stimulus type on $\gamma$ oscillatory power in our experiments, we first collapsed data collected on investigation of social and object stimuli and asked how CNO versus vehicle treatment affected slow and fast $\gamma$ power in each layer of CA1 (Figs. 3, 4). For slow $\gamma$ oscillations, power was significantly less following CNO treatment than following vehicle treatment for recordings obtained from SO $\left(t_{(7)}=\right.$ 2.55, $p=0.038 ; N=8$; Fig. $3 B$ ) and the pyramidal cell layer $\left(t_{(7)}=3.09, p=0.018 ; N=8\right.$; Fig. $\left.3 A, C\right)$ of $C A 1$, but not in recordings obtained from $S R\left(t_{(6)}=1.19, p=0.278 ; N=7\right.$; Fig. $3 D)$ or SLM $\left(t_{(6)}=0.34, p=0.745 ; N=7\right.$; Fig. $\left.3 E\right)$. For fast $\gamma$ oscillations, power was significantly less following CNO treatment than following vehicle treatment for recordings obtained from SO $\left(t_{(7)}=4.55, p=0.003 ; N=8\right.$; Fig. $3 B)$, the pyramidal cell layer $\left(t_{(7)}=7.36, p=0.0002\right.$; $N=8$; Fig. $3 C$ ) and SR $t_{(6)}=3.35, p=0.015 ; N=7$; Fig. $3 D$ ) but not for recordings obtained from SLM $\left(t_{(6)}=1.90\right.$, $p=0.107 ; N=7$; Fig. 3E).

We also measured theta powers and found no significant difference in theta power between CNO or vehicle treatment in Amigo2-icreERT2+ for recordings obtained from SO $\left(t_{(7)}=1.45, p=0.192\right.$; Fig. $\left.3 B\right)$, the pyramidal cell layer $\left(t_{(7)}=0.466, p=0.655\right.$; Fig. $\left.3 C\right)$, SR $\left(t_{(6)}=0.999, p=0.356\right.$; Fig. $\left.3 D\right)$, or $\operatorname{SLM}\left(t_{(6)}=1.52\right.$, $p=0.180$; Fig. $3 E)$.

In control Amigo2-icreERT2- mice, we found a significant main effect of layer $\left(F_{(1,3)}=24.04, p=0.000 ; N=7\right)$ and frequency $\left(F_{(1,1)}=57.98, p=0.001 ; N=7\right)$, as well as an interaction effect between layer and frequency $\left(F_{(1,3)}=\right.$ $25.11, p=0.001 ; N=7)$. We found no main effect of stimulus type or drug and no interactions involving stimulus type or drug (Table 1). Despite the absence of main or interaction effects of drug in Amigo2-icreERT2- mice, we compared each of slow and fast $\gamma$ powers for each layer of CA1 following versus vehicle treatment because identical analyses were performed in Amigo2-icreERT2+ mice.

For Amigo2-icreERT2- animals, slow $\gamma$ power was not significantly different following $\mathrm{CNO}$ or vehicle treatment for recordings obtained from SO $\left(t_{(5)}=0.74, p=0.493\right.$; $N=6$; Fig. $4 B$ ), the pyramidal cell layer $\left(t_{(6)}=0.33\right.$, $p=0.756 ; N=7 ;$ Fig. $4 A, C)$, SR $\left(t_{(6)}=0.49, p=0.643\right.$; $N=7$; Fig. $4 D)$, or SLM $\left(t_{(6)}=1.26, p=0.253 ; N=7\right.$; Fig. $4 E)$. Similarly, fast $\gamma$ power was not significantly different following $\mathrm{CNO}$ or vehicle treatment for recordings obtained from SO $\left(t_{(5)}=0.91, p=0.405 ; N=6\right.$; Fig. $\left.4 B\right)$, the 
pyramidal cell layer $\left(t_{(6)}=0.96, p=0.373 ; N=7\right.$; Fig. $\left.4 C\right)$, $\operatorname{SR}\left(t_{(6)}=0.59, p=0.579 ; N=7\right.$; Fig. $\left.4 D\right)$, or SLM $\left(t_{(6)}=67\right.$, $p=0.527 ; N=7 ;$ Fig. $4 E$ ).

Theta power was also not significantly different between CNO or vehicle treatment in Amigo2-icreERT2mice for recordings obtained from $S O\left(t_{(5)}=1.16\right.$, $p=0.299$; Fig. $4 B$ ), the pyramidal cell layer $\left(t_{(6)}=0.351\right.$, $p=0.737$; Fig. $4 C)$, SR $\left(t_{(6)}=0.438, p=0.677\right.$; Fig. $4 D$ ) or $\operatorname{SLM}\left(t_{(6)}=0.226, p=0.829\right.$; Fig. $\left.4 E\right)$.

We also compared mean velocity of the animals during the times used for LFP analysis for each treatment and stimulus presentation and found no significant differences in locomotor velocity for either Amigo2-icreERT2+ animals (main effect of stimulus: $F_{(1,7)}=0.000629, p=0.981$; main effect of treatment: $F_{(1,7)}=1.083, p=0.333$ ) or Amigo2-icreERT2- animals (main effect of stimulus: $F_{(1,6)}$ $=0.1282, p=0.733$; main effect of treatment: $F_{(1,6)}=$ $0.02,376, p=0.883)$.

\section{Inhibition of CA2 pyramidal cells reduces slow $\gamma$ power on social but not object stimulation}

Our a priori hypothesis before beginning this study was that inhibition of CA2 may alter $\gamma$ oscillatory power in a stimulus-specific manner. Therefore, as a follow-up analysis to that presented in Figures 3, 4, we separated $\gamma$ oscillatory power data according to stimulus and compared slow and fast $\gamma$ power upon either vehicle or CNO treatment for each type of stimulus exposure.

For Amigo2-icreERT2+ animals, we found that slow $\gamma$ power was significantly reduced in the pyramidal cell layer upon CNO treatment compared with vehicle treatment selectively during novel animal investigation; slow $\gamma$ power was not significantly reduced following CNO treatment during novel object investigation but was significantly reduced during novel animal investigation (main effect of treatment: $F_{(1,7)}=9.5, p=0.0178$; main effect of stimulus: $F_{(1,7)}=2.357 ; p=0.1686$; interaction: $F_{(1,7)}=$ 1.099, $p=0.3293$; novel animal: $p=0.0136$, novel object: $p=0.1063)$. In the SO layer, slow $\gamma$ power was overall significantly reduced following $\mathrm{CNO}$ treatment compared with vehicle treatment but each stimulus alone did not significantly reduce slow $\gamma$ power (main effect of treatment: $F_{(1,7)}=6.477, p=0.0384$; main effect of stimulus: $F_{(1,7)}=$ $0.5069 ; p=0.4995$; interaction: $F_{(1,7)}=0.4712, p=0.5145$; novel animal: $p=0.1015$, novel object: $p=0.3913)$. Slow $\gamma$ power was not significantly reduced by $\mathrm{CNO}$ in either SR (main effect of treatment: $F_{(1,6)}=1.416, p=0.2790$; main effect of stimulus: $F_{(1,6)}=0.08,567 ; p=0.7796$; interaction: $F_{(1,6)}=0.432, p=0.5354$ ) or SLM (main effect of treatment: $F_{(1,6)}=0.1542, p=0.71081$; main effect of stimulus: $F_{(1,6)}=0.02,751 ; p=0.837$; interaction: $F_{(1,6)}=0.2365$, $p=0.6440)$.

Similar to slow $\gamma$ power in the pyramidal cell layer, among Amigo2-icreERT2 + animals, fast $\gamma$ power in the pyramidal cell layer was also significantly reduced following CNO treatment. Interestingly though, whereas slow $\gamma$ power reduction was selective for social stimulus exposure, fast $\gamma$ power in the pyramidal cell layer was significantly reduced during both novel social stimulus and novel object stimulus exposure (main effect of treatment: $F_{(1,7)}=55.47, p=0.0001$; main effect of stimulus: $F_{(1,7)}=0.003674 ; p=0.9534$; interaction: $F_{(1,7)}=0.9945, p=0.3519$; novel animal: $p=0.0109$, novel object: $p=0.0021)$. In the SO and SR layers, fast $\gamma$ power was overall significantly reduced following CNO treatment compared with vehicle treatment (SO: main effect of treatment: $F_{(1,7)}=20.82, p=0.0026$; main effect of stimulus: $F_{(1,7)}=0.001639, p=0.9688$; interaction: $F_{(1,7)}=0.0263$, $p=0.8758$; SR: main effect of treatment: $F_{(1,6)}=11.2, p=$ 0.0155 ; main effect of stimulus: $F_{(1,6)}=0.1965, p=0.6731$; interaction: $\left.F_{(1,6)}=0.3173, p=0.5936\right)$ but each stimulus alone did not significantly reduce slow $\gamma$ power (SO: novel animal: $p=0.1303$; novel object: $p=0.1813$; SR: novel animal: $p=0.6322$; novel object: $p=0.2553$ ). Fast $\gamma$ power was not significantly reduced by CNO in SLM (main effect of treatment: $F_{(1,6)}=3.574, p=0.1076$; main effect of stimulus: $F_{(1,6)}$ $=1.022, p=0.3511$; interaction: $\left.F_{(1,6)}=4.355, p=0.0820\right)$.

Among Amigo2-icreERT2- animals (Fig. 6), we found no significant difference in slow $\gamma$ or fast $\gamma$ powers on either $\mathrm{CNO}$ or vehicle administration for recordings obtained from SO (slow $\gamma$ : main effect of treatment: $F_{(1,5)}=$ $0.5475, p=0.4926$; main effect of stimulus: $F_{(1,5)}=$ $0.01,439 p=0.9092$; fast $\gamma$ : main effect of treatment: $F_{(1,5)}$ $=0.8265, p=0.4050$; main effect of stimulus: $F_{(1,5)}=$ 2.188, $p=0.1992$ ), the pyramidal cell layer (slow $\gamma$ : main effect of treatment: $F_{(1,6)}=0.1057, p=0.7562$; main effect of stimulus: $F_{(1,6)}=0.02,277, p=0.8850$; fast $\gamma$ : main effect of treatment: $F_{(1,6)}=0.9247, p=0.3734$; main effect of stimulus: $F_{(1,6)}=1.999, p=0.2072$ ), SR (slow $\gamma$ : main effect of treatment: $F_{(1,6)}=0.2309, p=0.6479$; main effect of stimulus: $F_{(1,6)}=0.1713, p=0.6933$; fast $\gamma$ : main effect of treatment: $F_{(1,6)}=0.3515, p=0.5749$; main effect of stimulus: $\left.F_{(1,6)}=1.321, p=0.2941\right)$ or SLM (slow $\gamma$ : main effect of treatment: $F_{(1,6)}=1.616, p=0.2507$; main effect of stimulus: $F_{(1,6)}=2273, p=0.6504$; fast $\gamma$ : main effect of treatment: $F_{(1,6)}=0.4432, p=0.5303$; main effect of stimulus: $\left.F_{(1,6)}=0.2385, p=0.6426\right)$.

\section{CA2 pyramidal cell inhibition does not affect investigation time}

Based on our findings of reduced slow and fast $\gamma$ power on acute inhibition of CA2 pyramidal cells during investigation of novel stimuli, we asked whether the effects of CNO observed on neuronal oscillations were accompanied by changes in behavior that may have been responsible for changes in neuronal activity. First, we assessed whether CNO administration alone caused changes in the time subjects spent investigating the different types of stimuli. In Amigo2-icreERT2+ mice, CNO did not significantly affect the amount of time subjects spent investigating novel animals $\left(N=8 ; t_{(7)}=1.91\right.$, $p=0.098$; Fig. $7 A)$ or novel objects $\left(N=8 ; t_{(7)}=0.02\right.$, $p=0.981$ ). Similarly, in control Amigo2-icreERT2- mice, CNO did not cause a significant change in the amount of time subjects spent investigating novel animals $\left(N=7 ; t_{(6)}\right.$ $=0.32, p=0.761$; Fig. $7 A)$ or novel objects $\left(N=7 ; t_{(6)}=\right.$ $1.25, p=0.257$ ). Additionally, we assessed whether subjects showed a preference for either the novel animal or novel object stimulus following either vehicle or CNO administration based on time spent investigating the different stimulus types. Amigo2-icreERT2+ mice did not 
A

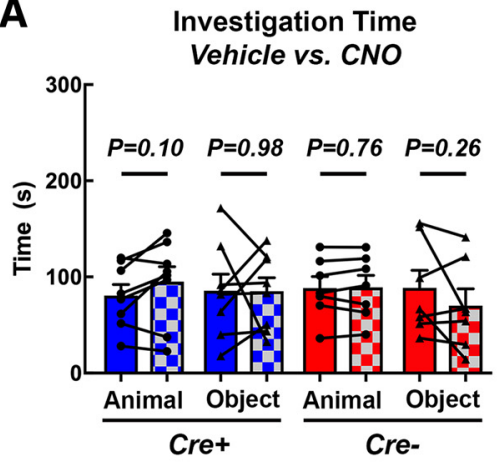

C

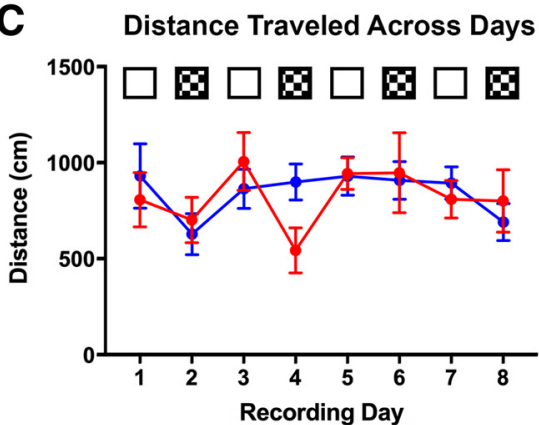

Investigation Time
Animal vs. Object

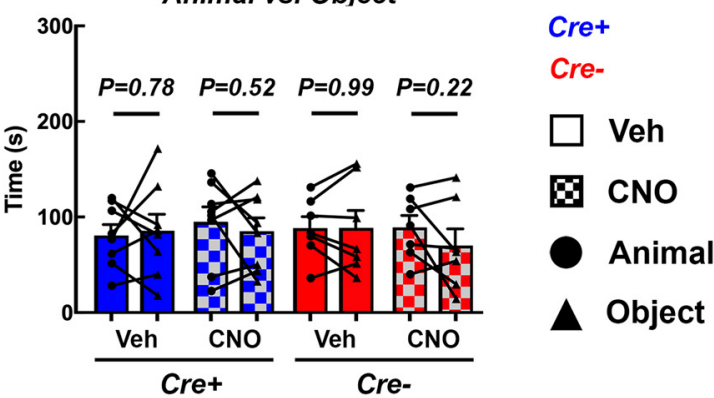

Time Mobile Across Days

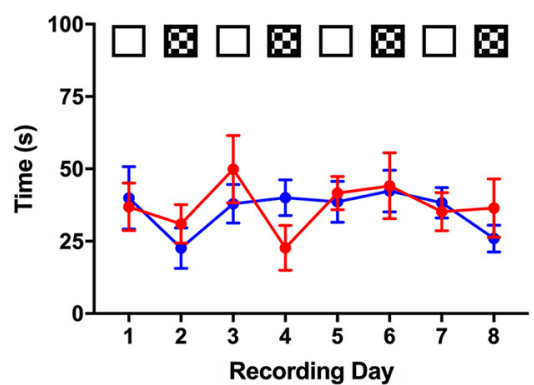

Figure 7. Effect of CNO on stimulus investigation time and activity level. $\boldsymbol{A}$, Time subject mice spent investigating different stimuli following administration of CNO or vehicle. CNO did not significantly affect the amount of time Amigo2-icreERT2+ or Amigo2icreERT2- mice spent investigating animals or objects. $\boldsymbol{B}$, Comparison of time spent investigating animals versus objects following either vehicle or CNO administration. Neither Amigo2-icreERT2 + nor Amigo2-icreERT2- mice exhibited a preference for one stimulus type over the other following vehicle or CNO administration. $\boldsymbol{C}, \boldsymbol{D}$, Mean distance traveled $(\boldsymbol{C})$ and time spent mobile $(\boldsymbol{D})$ measured across recording days for Amigo2-icreERT2 + (blue line) and Amigo2-icreERT2- (red line) mice. Mobile periods were based on a 7-cm/ $\mathrm{s}$ movement threshold with speeds greater than the threshold considered periods of mobility. Days on which CNO was administered are indicated by hash-marked bars or boxes, and days on which vehicle was administered are indicated by solid white or color bars or boxes. Amigo2-icreERT2 + cohorts are represented by red lines and bars and Amigo2-icreERT- cohorts are represented by blue lines and bars. For $\boldsymbol{A}, \boldsymbol{B}$, data points represent individual subject values and bars represent group averages. Circles indicate when an animal was used as the stimulus and triangles indicate when the stimulus was an object. Error bars indicate the SEM.

exhibit a preference for either stimulus type following vehicle $\left(N=8 ; t_{(7)}=0.29, p=0.784\right.$; Fig. $\left.7 B\right)$ or $\mathrm{CNO}(N=8$; $\left.t_{(7)}=0.67, p=0.521\right)$ administration. Similarly, control Amigo2-icreERT2- mice did not show a preference for either stimulus type following vehicle $\left(N=7 ; t_{(6)}=0.02\right.$, $p=0.985$; Fig. $7 B)$ or $\mathrm{CNO}\left(N=7 ; t_{(6)}=1.37, p=0.221\right) \mathrm{ad}-$ ministration. Additionally, we measured and found no difference in the average distance traveled across experimental days by Amigo2-icreERT2 $+\left(F_{(3.421,23.95)}=\right.$ 2.141, $p=0.1149$; Fig. 7C, blue line) or Amigo2-icreERT2mice $\left(F_{(3.027,18.16)}=1.772, p=0.1880\right.$; Fig. $7 C$, red line $)$. Finally, we found no difference in the average time spent mice mobile across experimental days by Amigo2icreERT2 $+\left(F_{(3.459,24.21)}=2.082, p=0.1219\right.$; Fig. $7 D$, blue line) or Amigo2-icreERT2- mice $\left(F_{(3.131,18.78)}=1.528\right.$, $p=0.2394$; Fig. $7 D$, red line). Therefore, the changes in slow and fast $\gamma$ powers on acute inhibition of CA2 are not likely to be due to changes in behavior.

\section{Discussion}

In this study, we recorded LFPs from across CA1 layers in adult, male Amigo2-icreERT2 mice and examined neuronal oscillations in the theta, slow $\gamma$ and fast $\gamma$ frequency ranges while subjects freely explored novel juvenile conspecifics or novel inanimate objects that were confined to a single position in space. We then chemogenetically inhibited CA2 pyramidal cells using hM4Di to determine whether CA2 contributes to the organization of theta, slow $\gamma$ or fast $\gamma$ oscillations in CA1 during investigation of novel stimuli. We found that acute inhibition of CA2 pyramidal cells reduced $\gamma$ power in a layer- and frequencydependent manner, with effects limited to the dendritic domains targeted by CA2 axons. In addition, analysis of changes in $\gamma$ power by each type of stimulus revealed a stimulus-specific effect of silencing CA2 on $\gamma$ oscillatory power; CA2 inhibition decreased slow $\gamma$ power in the pyramidal cell layer during investigation of novel animals but not novel objects. Interestingly, CA2 inhibition decreased fast $\gamma$ power in the pyramidal cell layer during both novel animal and novel object investigation. No significant change in $\gamma$ power was detected in SLM, the only layer not targeted by CA2 neurons. In addition, we observed no significant change in theta oscillatory power on CA2 inhibition. These findings reveal three novel points about the role of CA2 in organizing hippocampal oscillatory networks. 
First, CA2 modulates $\gamma$ activity in the SR and SO dendritic layers of CA1 in addition to the pyramidal cell layer but does not affect SLM. The generation of independent, layer-specific $\gamma$ oscillations in CA1 has previously been shown to depend on selective axonal targeting from upstream regions that modulate firing of CA1 GABAergic interneurons (Lasztóczi and Klausberger, 2014). CA2 pyramidal cells provide feed-forward inhibition as well as direct excitation of CA1 neurons (Valero et al., 2015; Boehringer et al., 2017), enabling it to modulate the balance of synaptic excitation and inhibition, a key feature of $\gamma$ oscillations. In addition to engaging interneurons within CA1, CA2 neurons target local inhibitory basket cells that project to CA1 (Mercer et al., 2012), providing another source of CA2-driven inhibition in CA1. Additionally, shifts in CA1 output have been shown to reflect discrete excitatory events generated by CA3 Schaffer collateral input at the $\gamma$ frequency, which serves as an accurate readout of the presynaptic population's activity level (FernándezRuiz et al., 2012). Thus, the observation that CA2 inhibition caused a selective reduction in $\gamma$ power in the dendritic domains targeted by CA2 axons suggests that CA2, and not some other CA1-projecting region, is responsible for the observed modulation of $\gamma$ power. These data support the idea that $\gamma$ oscillations facilitate communication between CA2 and CA1 in a manner similar to the CA3CA1 interface.

Second, these experiments suggest that in addition to regulating slow $\gamma$ oscillations, CA2 neuronal activity may serve to amplify fast $\gamma$ oscillations in CA1. Slow $\gamma$ oscillations in CA1 have previously been shown to rely on CA3 Schaffer collateral input (Middleton and McHugh, 2016). In addition, CA2 has been shown to contribute to the generation of slow $\gamma$ oscillations in the pyramidal cell layer of CA1 while subjects traverse an open field with no stimulus present (Alexander et al., 2018). Here, we confirm and extend those findings by demonstrating that the CA1 layers where slow $\gamma$ oscillations are affected by CA2 silencing match the layers to which CA2 neurons project. Fast $\gamma$ oscillations, by contrast, have been shown to rely on medial EC input to dentate gyrus and SLM (Bragin et al., 1995), and CA2 inhibition was previously not shown to decrease fast $\gamma$ while subject traversed an open field with no stimuli present (Alexander et al., 2018). By contrast, our findings demonstrate a role for CA2 activity in fast $\gamma$ oscillations in CA1 during immobile investigation of novel stimuli in that silencing of CA2 output decreased fast $\gamma$ power in SO, SR and the pyramidal cell layer. One possible explanation for this finding is that CA2 activity may normally facilitate fast $\gamma$ oscillation propagation within the hippocampal circuitry during investigation of novel stimuli (animal, object, or otherwise), and bilateral silencing of CA2 may remove this faciliatory role of CA2.

Third, we provide evidence that slow $\gamma$ oscillations are decreased in response to CA2 silencing during investigation of novel social stimuli but not novel object stimuli. CA2 has been shown to be required for social recognition memory (Hitti and Siegelbaum, 2014), but whether CA2 neurons coordinate their activity with CA1 when processing stimuli containing socially relevant information remained unexplored. We predicted that if CA2 is preferentially involved in processing information about socially relevant stimuli, then CA2 pyramidal cells may organize rhythmic activity in CA1 only during investigation of an animal and not an object such that silencing of CA2 activity would impact oscillatory activity preferentially during investigation of social stimuli. Further, based on the layer-specificity of CA2 axons within CA1, we expected that any altered oscillatory activity would be observed selectively in CA2-projection layers. Indeed, we found that $\gamma$ oscillatory power recorded from a CA2-projection layer was affected by CA2 inhibition during social investigation.

Given the previous finding that chemogenetic modulation of CA2 neuronal activity affects slow $\gamma$ power in the CA1 pyramidal cell layer (Alexander et al., 2018), we favored the hypothesis that slow $\gamma$ power but not high $\gamma$ power would be decreased by CA2 silencing during novel animal investigation. Accordingly, we found that slow $\gamma$ power was reduced on CA2 inhibition. Somewhat surprisingly though, we also found that fast $\gamma$ power was reduced on CA2 inhibition and it was reduced during both novel animal and novel object investigation. CA2 neuronal activity patterns have been shown to change (i.e., place cell remapping) in response to novel conspecifics and to novel objects (Alexander et al., 2016), and CA2 neurons have been shown to respond to novelty even when there is no social component involved in the task (Wintzer et al., 2014; Lu et al., 2015), so it is not surprising that novel object investigation as well as novel animal investigation would engage CA2 neuronal activity. Possibly, slow $\gamma$ oscillations originating from activity of $\mathrm{CA} 2$ and $\mathrm{CA} 3$ neurons are engaged by social investigation, whereas fast $\gamma$ oscillations originating from activity of medial EC neurons and propagated by way of the dentate gyrus to hippocampus in response to investigation of any novel stimulus (Kemere et al., 2013; Zheng et al., 2016), are facilitated by the activity of CA2 neurons.

Based on our physiological findings, we asked whether the observed reductions in $\gamma$ power were accompanied by changes in behavior that may have produced the changes in neuronal oscillations. We measured time spent investigating the different types of stimuli following treatment with $\mathrm{CNO}$ or vehicle and found no difference in the amount of time mice spent investigating either stimulus type following either treatment. Because we were interested in the role of CA2 in hippocampal oscillatory networks and the electrophysiological effects of targeted CA2 manipulation, we selected a behavior that did not require behavioral training. For this reason, the lack of a significant effect of CA2 inhibition on stimulus investigation times is not surprising, as we used a passive exposure paradigm in which subjects could freely explore the environment of their own volition, with each session separated by $24 \mathrm{~h}$. In contrast, social recognition paradigms typically measure investigation time of social stimuli on repeated exposures taking place over the course of minutes to hours (Hitti and Siegelbaum, 2014; Smith et al., 2016). These differences in experimental design do not necessarily suggest that the electrophysiological effects described here are not relevant to behavior, but that a behavioral paradigm 
explicitly designed to assess changes in memory would be required to reveal their behavioral relevance. Further, the lack of behavioral changes in our study makes the interpretation of electrophysiological data more straightforward, as differences in behavior can also cause changes in oscillatory activity and confound whether observed effects are due to experimental manipulation or the different behaviors performed.

In addition, we note that the dose of CNO we used $(3 \mathrm{mg} / \mathrm{kg}$ ) was lower than that used in previous study of CA2 and social memory $(10 \mathrm{mg} / \mathrm{kg}$; Meira et al., 2018). However, a lower dose of $5 \mathrm{mg} / \mathrm{kg} \mathrm{CNO}$ was previously shown to silence CA2-driven responses in CA1 by $>95 \%$ (Alexander et al., 2018), and a dose of $4 \mathrm{mg} / \mathrm{kg} \mathrm{CNO}$ has been shown to effectively inhibit social memory by inhibiting ventral CA3 neuron activity (Chiang et al., 2018). Therefore, although not examined in the current study, we would expect that the CNO dose used in the current study would impair social recognition memory and is appropriate for study of neuronal activity patterns that may be involved in social memory.

In conclusion, we found that CA2 neuronal activity contributes to slow and fast $\gamma$ oscillations in CA1 in a dendritic layer-dependent manner. Slow $\gamma$ oscillations were preferentially impacted by CA2 silencing during novel animal investigation over novel object investigation, whereas fast $\gamma$ oscillations were affected by CA2 silencing during either novel animal or novel object investigation. These findings support a role of CA2 neuronal activity in hippocampal $\gamma$ oscillations and processing of social and contextual information.

\section{References}

Alexander GM, Farris S, Pirone JR, Zheng C, Colgin LL, Dudek SM (2016) Social and novel contexts modify hippocampal CA2 representations of space. Nat Commun 7:10300.

Alexander GM, Brown LY, Farris S, Lustberg D, Pantazis C, Gloss B, Plummer N, Jensen P, Dudek SM (2018) CA2 neuronal activity controls hippocampal low gamma and ripple oscillations. Elife 7: e38052.

Boehringer R, Polygalov D, Huang AJY, Middleton SJ, Robert V, Wintzer ME, Piskorowski RA, Chevaleyre V, McHugh TJ (2017) Chronic loss of CA2 transmission leads to hippocampal hyperexcitability. Neuron 94:642-655.e9.

Bragin A, Jandó G, Nádasdy Z, Hetke J, Wise K, Buzsáki G (1995) Gamma (40-100 Hz) oscillation in the hippocampus of the behaving rat. J Neurosci 15:47-60.

Buzsáki G, Draguhn A (2004) Neuronal oscillations in cortical networks. Science 304:1926-1929.

Buzsáki G, Buhl DL, Harris KD, Csicsvari J, Czéh B, Morozov A (2003) Hippocampal network patterns of activity in the mouse. Neuroscience 116:201-211.

Chiang MC, Huang AJY, Wintzer ME, Ohshima T, McHugh TJ (2018) A role for CA3 in social recognition memory. Behav Brain Res 354:22-30.

Chevaleyre V, Siegelbaum SA (2010) Strong CA2 pyramidal neuron synapses define a powerful disynaptic cortico-hippocampal loop. Neuron 66:560-572.

Colgin LL (2015) Do slow and fast gamma rhythms correspond to distinct functional states in the hippocampal network? Brain Res 1621:309-315.

Colgin LL (2016) Rhythms of the hippocampal network. Nat Rev Neurosci 17:239-249.
Colgin LL, Denninger T, Fyhn M, Hafting T, Bonnevie T, Jensen O, Moser MB, Moser El (2009) Frequency of gamma oscillations routes flow of information in the hippocampus. Nature 462:353357.

Csicsvari J, Hirase H, Czurkó A, Mamiya A, Buzsáki G (1999) Fast network oscillations in the hippocampal CA1 region of the behaving rat. J Neurosci 19:RC20.

Dudek SM, Alexander GM, Farris S (2016) Rediscovering area CA2: unique properties and functions. Nat Rev Neurosci 17:89-102.

Fernández-Ruiz A, Makarov VA, Benito N, Herreras O (2012) Schaffer-specific local field potentials reflect discrete excitatory events at gamma frequency that may fire postsynaptic hippocampal CA1 units. J Neurosci 32:5165-5176.

Hitti FL, Siegelbaum SA (2014) The hippocampal CA2 region is essential for social memory. Nature 508:88-92.

Kay K, Sosa M, Chung JE, Karlsson MP, Larkin MC, Frank LM (2016) A hippocampal network for spatial coding during immobility and sleep. Nature 531:185-190.

Kemere C, Carr MF, Karlsson MP, Frank LM (2013) Rapid and continuous modulation of hippocampal network state during exploration of new places. PLoS One 8:e73114.

Kohara K, Pignatelli M, Rivest AJ, Jung HY, Kitamura T, Suh J, Frank D, Kajikawa K, Mise N, Obata Y, Wickersham IR, Tonegawa S (2014) Cell type-specific genetic and optogenetic tools reveal hippocampal CA2 circuits. Nat Neurosci 17:269-279.

Laeremans A, Nys J, Luyten W, D'Hooge R, Paulussen M, Arckens L (2013) AMIGO2 mRNA expression in hippocampal CA2 and CA3a. Brain Struct Funct 218:123-130.

Lasztóczi B, Klausberger T (2014) Layer-specific GABAergic control of distinct gamma oscillations in the CA1 hippocampus. Neuron 81:1126-1139.

Lasztóczi B, Klausberger T (2016) Hippocampal place cells couple to three different gamma oscillations during place field traversal. Neuron 91:34-40.

Lee SE, Simons SB, Heldt SA, Zhao M, Schroeder JP, Vellano CP, Cowan DP, Ramineni S, Yates CK, Feng Y, Smith Y, Sweatt JD, Weinshenker D, Ressler KJ, Dudek SM, Hepler JR (2010) RGS14 is a natural suppressor of both synaptic plasticity in CA2 neurons and hippocampal-based learning and memory. Proc Natl Acad Sci USA 107:16994-16998.

Lein ES, Hawrylycz MJ, Ao N, Ayres M, Bensinger A, Bernard A, Boe AF, Boguski MS, Brockway KS, Byrnes EJ, Chen L, Chen L, Chen TM, Chin MC, Chong J, Crook BE, Czaplinska A, Dang CN, Datta S, Dee NR, et al. (2007) Genome-wide atlas of gene expression in the adult mouse brain. Nature 445:168-176.

Lu L, Igarashi KM, Witter MP, Moser El, Moser MB (2015) Topography of place maps along the CA3-to-CA2 axis of the hippocampus. Neuron 87:1078-1092.

Mankin EA, Diehl GW, Sparks FT, Leutgeb S, Leutgeb JK (2015) Hippocampal CA2 activity patterns change over time to a larger extent than between spatial contexts. Neuron 85:190-201.

Mercer A, Eastlake K, Trigg HL, Thomson AM (2012) Local circuitry involving parvalbumin-positive basket cells in the CA2 region of the hippocampus. Hippocampus 22:43-56.

Meira T, Leroy F, Buss EW, Oliva A, Park J, Siegelbaum SA (2018) A hippocampal circuit linking dorsal CA2 to ventral CA1 critical for social memory dynamics. Nat Commun 9:4163.

Middleton SJ, McHugh TJ (2016) Silencing CA3 disrupts temporal coding in the CA1 ensemble. Nat Neurosci 19:945-951.

Oliva A, Fernández-Ruiz A, Buzsáki G, Berényi A (2016) Role of hippocampal $\mathrm{CA} 2$ region in triggering sharp-wave ripples. Neuron 91:1342-1355.

San Antonio A, Liban K, Ikrar T, Tsyganovskiy E, Xu X (2014) Distinct physiological and developmental properties of hippocampal CA2 subfield revealed by using anti-Purkinje cell protein 4 (PCP4) immunostaining. J Comp Neurol 522:1333-1354.

Scheffer-Teixeira R, Belchior H, Caixeta FV, Souza BC, Ribeiro S, Tort AB (2012) Theta phase modulates multiple layer-specific oscillations in the CA1 region. Cereb Cortex 22:2404-2414. 
Schomburg EW, Fernández-Ruiz A, Mizuseki K, Berényi A, Anastassiou CA, Koch C, Buzsáki G (2014) Theta phase segregation of input-specific gamma patterns in entorhinal-hippocampal networks. Neuron 84:470-485.

Smith AS, Williams Avram SK, Cymerblit-Sabba A, Song J, Young WS (2016) Targeted activation of the hippocampal CA2 area strongly enhances social memory. Mol Psychiatry 21:1137-1144.

Stevenson EL, Caldwell HK (2012) The vasopressin 1b receptor and the neural regulation of social behavior. Horm Behav 61:277-282.

Tamamaki N, Abe K, Nojyo Y (1988) Three-dimensional analysis of the whole axonal arbors originating from single CA2 pyramidal neurons in the rat hippocampus with the aid of a computer graphic technique. Brain Res 452:255-272.
Tulving E (1984) Precis of elements of episodic memory. Behav Brain Sci 7:223-268.

Valero M, Cid E, Averkin RG, Aguilar J, Sanchez-Aguilera A, Viney TJ, Gomez-Dominguez D, Bellistri E, de la Prida LM (2015) Determinants of different deep and superficial CA1 pyramidal cell dynamics during sharp-wave ripples. Nat Neurosci 18:1281-1290.

Wintzer ME, Boehringer R, Polygalov D, McHugh TJ (2014) The hippocampal CA2 ensemble is sensitive to contextual change. J Neurosci 34:3056-3066.

Zheng C, Bieri KW, Hwaun E, Colgin LL (2016) Fast gamma rhythms in the hippocampus promote encoding of novel object-place pairings. eNeuro 3:ENEURO.0001-16.2016. 